\title{
Perspective
}

PERSPECTIVE Actualité en histoire de l'art

Comptes rendus | 2015

\section{Aziza Gril-Mariotte, Les toiles de Jouy : histoire d'un art décoratif, 1760-1821, Étienne Jollet (préface), Rennes, Presses universitaires de Rennes (Arts et Société), 2015.}

Rossella Froissart

\section{(2) OpenEdition \\ Journals}

Édition électronique

URL : http://journals.openedition.org/perspective/6610

DOI : $10.4000 /$ perspective.6610

ISSN : 2269-7721

Éditeur

Institut national d'histoire de l'art

\section{Référence électronique}

Rossella Froissart, "Aziza Gril-Mariotte, Les toiles de Jouy : histoire d'un art décoratif, 1760-1827, Étienne Jollet (préface), Rennes, Presses universitaires de Rennes (Arts et Société), 2015. », Perspective [En ligne], Comptes rendus, mis en ligne le 28 juillet 2016, consulté le 01 octobre 2020. URL : http:// journals.openedition.org/perspective/6610 ; DOI : https://doi.org/10.4000/perspective.6610 


\section{Aziza Gril-Mariotte, Les toiles de Jouy : histoire d'un art décoratif, 1760-1821, Étienne Jollet (préface), Rennes, Presses universitaires de Rennes (Arts et Société), 2015.}

Rossella Froissart

\section{RÉFÉRENCE}

Aziza Gril-Mariotte, Les toiles de Jouy : histoire d'un art décoratif, 1760-1821, Étienne Jollet (préface), Rennes, Presses universitaires de Rennes (Arts et Société), 2015. 
1 C'est d'abord à une fête de formes et de couleurs que nous convie A. Gril-Mariotte dans l'étude qu'elle consacre à l'une des entreprises économiques et artistiques les plus extraordinaires de la France contemporaine, celle dirigée par Christophe-Philippe Oberkampf entre 1760 et 1821 . Mais cet ouvrage ne vaut pas que pour le charme irrésistible dégagé par les nombreuses reproductions de textiles, dessins et « empreintes » qui illustrent le propos de l'auteur et qui ne sont qu'une infime partie de la documentation lui ayant servi de point de départ. A. GrilMariotte a fait de la masse imposante d'archives laissée par la fabrique de Jouy-

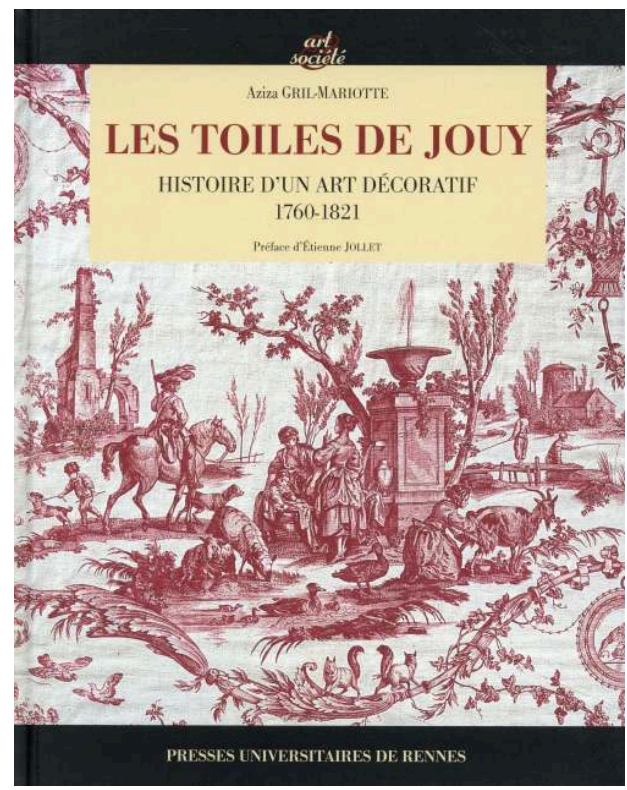
en-Josas, et dispersée aujourd'hui entre plusieurs institutions, le socle bien solide de l'histoire d'un art dont elle a patiemment reconstitué les évolutions aussi bien techniques qu'esthétiques. Tout en s'inscrivant dans le sillage du travail déjà accompli par les historiens sur la manufacture et sur son fondateur, dans la droite ligne d'une histoire sociale, économique et culturelle, A. Gril-Mariotte rend à cette production semi-industrielle ou industrielle de saynètes, figures et éléments décoratifs en tout genre, le statut de création artistique à part entière. Que celle-ci ait intégré la contrainte - l'outillage et les savoir-faire, la demande de la clientèle, les aléas historiques - ne diminue en rien la valeur du résultat final, dont le trait principal réside dans l'équilibre trouvé entre le déploiement d'une exceptionnelle inventivité et la pleine adéquation avec l'époque.

Dans une première partie introductive, l'auteur examine l'ensemble de la production de la manufacture à la lumière de l'évolution des techniques, des mutations du goût et des évolutions du statut des artistes et des ouvriers d'art employés. Les processus de fabrication des motifs sont aussi reconstitués dans toute leur variété : de l'invention pure et simple à la copie littérale - les deux également rares - en passant par l'imitation à partir d'autre supports - soieries, peinture, gravure, parfois porcelaine ou tapisserie -, transpositions, compositions par adjonction, collages ou arrangements. Les transferts d'Angleterre, fréquents au début, laissent la place dans les années 1770 à une grande liberté, pour donner lieu, avec l'arrivée de Jean-Baptiste Huet en 1783, à l'invention, exigée par Oberkampf lui-même, d'un "nouveau goût ", celui auquel le profane identifie encore aujourd'hui les toiles de Jouy. Néanmoins il n'est pas question pour A. Gril-Mariotte de centrer son propos sur l'intervention de ce peintre, ou de celle, à partir de 1813, de l'architecte Louis Hippolyte Lebas, car la peinture et la gravure ne sont que l'une des sources innombrables de la conception des formes. Dans les trois parties qui suivent, l'auteur déroule plus lentement le fil chronologique pour mieux approfondir la part de tradition présente dans l'innovation, les modes d'appropriation de l'exotisme - indiennages, chinoiseries et perses -, les penchants naturalistes ou, à l'opposé, ceux conduisant à la stylisation et à l'abstraction, l'influence de la peinture de genre et celle des recueils néo-classiques. Ces glissements d'une manière à l'autre ne sont nullement anodins et leur réussite est liée parfois au défis techniques 
qu'Oberkampf relève avec succès: la gravure au cylindre, les procédés chimiques d'impression par réserve, la taille des planches et la netteté de la taille, la solidité des teintes (le vert). Dans une dernière section A. Gril-Mariotte s'arrête sur les modes d'assemblage et sur le fonctionnement des images propre aux textiles à motifs historiques, d'actualité ou d'inspiration littéraires, en insistant sur l'influence de la peinture et sur les écarts passionnants des mécanismes de la vision entre le tableau et la toile imprimée.

Classées, datées, identifiées, les toiles de Jouy peuvent désormais déborder le seul champ, cher à Walter Benjamin, de la " perception distraite ». L'ampleur des sources et les nombreuses pistes ouvertes par A. Gril-Mariotte laissent espérer que l'exploration de cette histoire des formes produites au croisement de l'unicité et de la multiplicité, de l'invention technique et esthétique, se poursuivra dans des enquêtes nouvelles, s'élargissant à d'autres arts industriels. 